14.02

\title{
МРТ-управляемая терапия
}

\author{
() Ю.В. Богачев, ${ }^{1}$ А.В. Никитина, ${ }^{1}$ В.В. Фролов, ${ }^{2}$ В.И. Чижик ${ }^{2}$
}

${ }^{1}$ Санкт-Петербургский государственный электротехнический университет „ЛЭТИ“, 197376 Санкт-Петербург, Россия

${ }^{2}$ Санкт-Петербургский государственный университет, 199034 Санкт-Петербург, Россия e-mail: Yu.Bogachev@mail.ru

Поступило в Редакцию 21 января 2020 г.

В окончательной редакции 21 января 2020 г.

Принято к публикации 17 февраля 2020 г.

Магнитно-резонансная тераностика - новое направление современной медицины, в котором высокие диагностические возможности методов магнитного резонанса сочетаются с терапевтическим воздействием на различные органы физических полей разнообразной природы. Рассмотрены особенности и клинические применения методов и аппаратуры МР-тераностики, включающих МРТ-управляемые высокоинтенсивную фокусированную ультразвуковую хирургию (абляцию опухолей), радиационную (рентгеновскими лучами) терапию, протонную терапию, радиочастотную терапию.

Ключевые слова: магнитный резонанс, тераностика, магнитно-резонансная томография.

DOI: $10.21883 /$ JTF.2020.09.49680.22-20

\section{Введение}

Одним из признанных методов современной неинвазивной медицинской диагностики является магнитно-резонансная томография (МРТ) [1-4]. Магнитно-резонансная (МР) тераностика (сочетание слов „терапия“ и „диагностика“ - от греч. therapeia и diagnostikos) является новым, интенсивно-развивающимся направлением, в котором высокие диагностические возможности ме- тода МРТ сочетаются с терапевтическим воздействием на организм с помощью других физических методов или веществ. В настоящей работе рассматриваются методы МР-тераностики, уже нашедшие клиническое применение или находящиеся в стадии подготовки к клиническому апробированию, в которых терапевтическое воздействие различными физическими полями происходит под управлением МРТ. Эти методы включают МРТ-управляемые высокоинтенсивную фокусированную
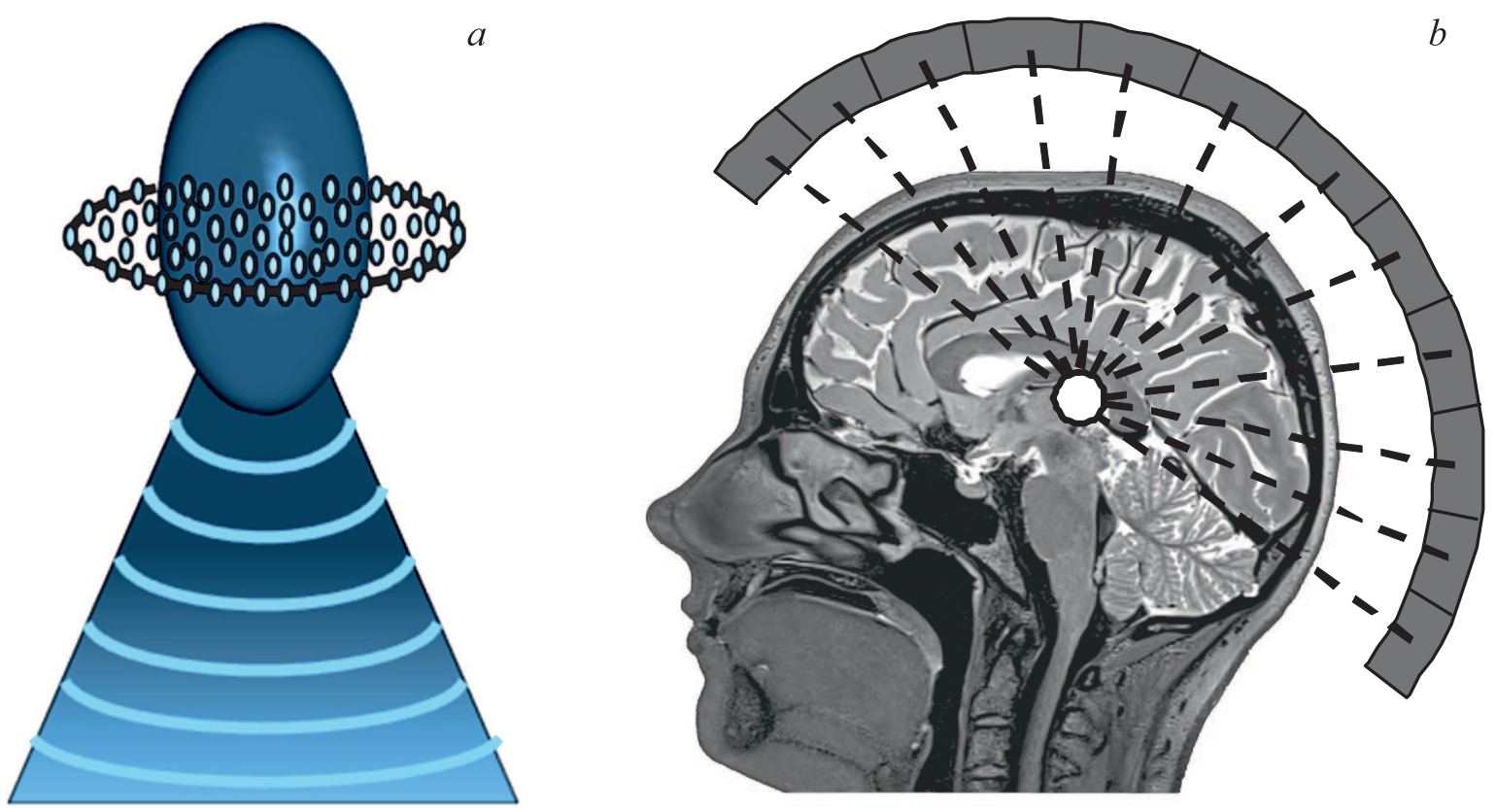

Pис. 1. $a-$ формирование зон абляции („спотов“) при MR-HIFU; $b$ - точечное воздействие фокусированным ультразвуком на проводящие пути в головном мозге. 
ультразвуковую хирургию (абляцию опухолей), радиационную (рентгеновскими лучами) терапию, протонную терапию, радиочастотную терапию.

В стадии исследований находятся новые методы МР-тераностики, такие как транскраниальная магнитная стимуляция, совмещенная с функциональной МРТ (TMS/fMRI) [5], MPT-управляемая фотодинамическая терапия [6,7], МР-управляемая терапия с помощью магнитных наночастиц [8-11]. Число таких исследований непрерывно растет и соответственно возрастает роль МР-тераностики в современной медицине.

\section{1. МРТ-управляемая высокоинтенсивная фокусированная ультразвуковая хирургия (абляция опухолей)}

Объединение метода высокоинтенсивной фокусированной ультразвуковой хирургии с магнитно-резонансной томографией позволило проводить неинвазивную абляцию опухолей с ориентацией на магнитно-резонансное изображение (в литературе встречаются два названия этого метода - MR-guided focused ultrasound surgery, сокращенно MRgFUS, и MR-guided high intensity ultrasound ablation, сокращенно MR-HIFU) [12].

Основным терапевтическим механизмом HIFU-абляции является доставка тепловой энергии к патологическому очагу с помощью фокусированного ультразвукового луча. Ультразвуковой луч генерируется высокомощным ультразвуковым преобразователем. Фокусировка луча достигается с помощью фазовой решетки ультразвукового преобразователя, выполненной в форме чаши, встраиваемой в столешницу MPT-сканера.

Первоначально абляция методом MR-HIFU проводилась от точки к точке: весь объем опухоли обрабатывался путем последовательного нацеливания на множество небольших очагов диаметром несколько миллиметров. Этот метод не только требовал много времени, но и был малоэффективен, поскольку большая часть поглощенной энергии терялась в результате рассеивания тепла после каждой точечной абляции. По этой причине был внедрен метод объемной MR-HIFU абляции, использующий электронное управление лучом с помощью фазированной решетки преобразователей, которые управляются сигналами, имеющими надлежащую разность фаз, чтобы сконцентрировать энергию в общей фокальной точке (электронная фокусировка) [13-15]. При объемной MR-HIFU-абляции фокус ультразвукового луча направляется вдоль точек по круговой или спиральной траектории, перпендикулярной ультразвуковому лучу (рис. $1, a)$. Быстрое $(\sim 50 \mathrm{~ms}$ переключение между различными точками фокусировки обеспечивает равномерный нагрев указанного объема опухоли или области обработки. Начиная с середины области обработки, диффузия тепла эффективно используется для нагрева всей области обработки.
Луч HIFU проникает в кожу и другие мягкие ткани, не вызывая их повреждения, но ткань в фокальной области луча (область порядка нескольких миллиметров) поглощает значительно больше акустической энергии, чем соседние области, что приводит к повышению температуры в зоне фокуса до $(60-90)^{\circ} \mathrm{C}[16]$. Нагрев происходит с таким высоким градиентом температуры, что на расстоянии $3 \mathrm{~mm}$ от фокуса температура заметно не повышается, а на расстоянии $1 \mathrm{~mm}$ поднимается всего на несколько градусов. Поэтому вне зоны фокуса (на всем пути прохождения ультразвуковых волн и в окружающих тканях) ультразвук не оказывает неблагоприятного воздействия. При нагревании ткани до температуры $54^{\circ} \mathrm{C}$ в течение $3 \mathrm{~s}$ (при температуре $60^{\circ} \mathrm{C}$ почти мгновенно) происходит денатурация белков и гибель всех клеток в облучаемой фокальной области. Один импульс HIFU продолжается в среднем $\sim 20 \mathrm{~s}$, что достаточно для термической коагуляции ткани. Зона коагуляции в результате одного импульса HIFU (так называемый „спот“) имеет форму эллипсоида (рис. 1, $a$ ) диаметром от 1 до $10 \mathrm{~mm}$ и длиной от 2 до $70 \mathrm{~mm}$. Комбинированием нескольких импульсов можно воздействовать на опухоль любого размера и любой формы.

Магнитно-резонансная томография (МРТ) в данном случае используется как гид, направляющий ультразвуковой луч по изображению. Это обеспечивает врачу высококачественные анатомические и физиологические данные в режиме реального времени и позволяет осуществлять эффективный мониторинг лечения.

Для абляции методом MR-HIFU обязательна термометрия в реальном масштабе времени и контроль распределения энергии в зоне воздействия, потому что температура нагрева не должна превышать $90^{\circ} \mathrm{C}$ (чтобы не было закипания межклеточной жидкости и повреждения ткани, пограничной к зоне абляции). Результаты термометрии в методе MR-HIFU отображаются в виде термограмм, окрашенных в определенные цвета в соответствии с температурой ткани. Термограммы создаются через каждые $3 \mathrm{~s}$ во время импульса HIFU и накладываются на МРТ-изображение обрабатываемой области. Участки, где температура превысила „порог коагуляции“, выделяются красным цветом на фоне чернобелого МРТ-изображения. Картирование температуры с помощью МРТ предоставляет врачу пространственновременную температурную информацию в режиме реального времени, которая может быть использована для мониторинга лечения в процессе абляции [17-19].

Наиболее широко используемым методом МР-картирования является метод химического сдвига частоты протонного резонанса (PRFS) [19]. Этот метод основан на температурной зависимости константы электронного экранирования ядер водорода (протонов) в воде. Этот эффект приводит к смещению наблюдаемой резонансной частоты протонов в водосодержащих тканях при изменении температуры и может быть использован для измерения температуры с точностью до $0.5^{\circ} \mathrm{C}$ in vitro. Точность измерений in vivo зависит от множества факторов, 
таких как плотность протонов в тканях, времена ЯМРрелаксации, движение органов, конструкция приемной катушки, размер вокселя, поле зрения и требуемое временное разрешение [17-19]. Временное разрешение для клинических применений обычно составляет от 2 до $6 \mathrm{~s}$ и зависит, главным образом, от количества томографических срезов, получаемых в целевой области, и мониторинга нагрева в ближнем и дальнем полях ультразвукового луча. Кроме того, использование анатомической МРТ обеспечивает прекрасное контрастирование мягких тканей для выявления границ опухолей на стадии планирования лечения.

Химический сдвиг частоты резонанса протонов является единственным параметром, определяемым исключительно по частоте резонансного сигнала, в отличие от других параметров, определяемых по амплитуде сигнала. Следовательно, протонный химический сдвиг может быть обнаружен независимо от других параметров, хотя он зависит от $\mathrm{pH}$ и присутствия ионов. Температурная зависимость химического сдвига протонов воды является с высокой корреляцией линейной в температурном диапазоне $(20-80)^{\circ} \mathrm{C}$. Температурный коэффициент сдвига резонансной частоты протонов воды в отличие от температурных коэффициентов других параметров, таких как время спин-решеточной релаксации, время спин-спиновой релаксации, плотность протонов и коэффициент диффузии, практически не зависит от вида ткани, находясь в диапазоне от 0.00739 до $0.0135 \mathrm{ppm} /{ }^{\circ} \mathrm{C}$. Вышеизложенное объясняет превосходство использования протонного химического сдвига частоты резонанса протонов воды в качестве индикатора температуры по сравнению с другими методами, основанными на сигнале ЯМР воды ${ }^{1} \mathrm{H}$.

Информация о температуре, полученная с помощью МР-картирования, может быть использована для оценки объема обрабатываемой ткани двумя способами. Вопервых, как известно, коагуляционный некроз возникает при температурах выше $57^{\circ} \mathrm{C}$, поэтому любая часть ткани, которая была нагрета до температуры выше $57^{\circ} \mathrm{C}$, может рассматриваться как некротическая. Второй, часто используемой величиной, отражающей нанесенное тепловое повреждение, является тепловая доза. Тепловая доза выражается в эквивалентных минутах при температуре $43^{\circ} \mathrm{C}\left(\mathrm{CEM}_{43}\right)$ [20,21]. Предклинические исследования показали, что тепловая доза, необходимая для некроза, составляет от 50 до $240 \mathrm{CEM}_{43}$ (в зависимости от типа ткани и вида исследования [20-22]), поэтому $240 \mathrm{CEM}_{43}$ часто используется в качестве предела летальной тепловой дозы при количественном определении площади некротической ткани. Соотношение между тепловой дозой и площадью некротической ткани in vivo носит оценочный характер, поскольку, например, вторичная гибель клеток, на развитие которой может уйти от нескольких часов до нескольких дней (например, из-за отеков или гипоперфузии), не принимается во внимание $[23,24]$. Для характеристики этих процессов необходимы дальнейшие исследования, включая комплексное наблюдение за подвергшимися абляции опухолями и анализ гистологических образцов.

Данные МРТ, описывающие пространственно-временное распределение температуры и аккумулированную термическую дозу, обеспечивают надежный и эффективный контроль применения ультразвуковой энергии, позволяют в ходе процедуры подстраивать параметры воздействия в соответствии со специфическими характеристиками обрабатываемой ткани, влияющими на степень ее нагрева. „Дозиметрическая карта“ процедуры позволяет контролировать правильность выполнения процедуры. Сразу по завершении процедуры проводится контрольное МРТ-сканирование с введением контрастирующего агента, позволяющее визуализировать участки отсутствия поглощения контраста, что указывает на реваскуляризацию и некроз ткани, т. е. „зоны без перфузии“. Эффективность процедуры MR-HIFU оценивается путем измерения объема „зоны без перфузии“, появившейся в обработанной опухоли.

Первые клинические применения метода MR-HIFU были реализованы с помощью системы „ExAblate“ фирмы INSIGHTEC (Израиль), интегрированной в единый комплекс с магнитно-резонансным томографом фирмы General Electric с индукцией магнитного поля $1.5 \mathrm{~T}$ для неинвазивной абляции опухолей. Процедура MR-HIFU не оказывает клинически значимого общего действия на организм, не требует госпитализации, наркоза, реабилитации и имеет огромные преимущества перед другими методами лечения, поскольку позволяет избежать инвазии, кровопотери, косметических дефектов и серьезных осложнений от хирургической операции и наркоза.

Основными клиническими применениями системы „ExAblate“ в настоящее время является абляция миомы матки, паллиативная абляция костных метастазов и абляция рака молочной железы [25,26], в ближайшей перспективе возможна абляция злокачественных опухолей головного мозга, печени, простаты и селезенки [27-29].

Помимо абляции опухолей метод MR-HIFU в последнее время начал применяться для обратимого преодоления гематоэнцефалического барьера с целью прицельной доставки лекарственных препаратов или генной терапии, для воздействия на дуго-отростчатые суставы позвонков с целью лечение болей в позвоночнике, для внутрисосудистого удаления тромбов при лечении инсульта (сонотромболизис) [30].

Благодаря технологии MR-HIFU появилась новая медицинская дисциплина - неинвазивная функциональная нейрохирургия [30], в основе которой лежит точечное воздействие фокусированным ультразвуком на проводящие пути в головном мозге (рис. 1,b). Фирмой INSIGHTEC (Израиль) разработана система „ЕxAblate Neuro“ для неинвазивного нейрохирургического транскраниального лечения людей, страдающих от тяжелых нейрогенных болей, двигательных расстройств типа эссенциального тремора, болезни Паркинсона и эпилепсии. Для пациентов с эссенциальным тремором и болезнью Паркинсона энергия ультразвука доставляется 
к вентральному промежуточному ядру таламуса через череп без разреза или краниотомии. Во время обработки высокоинтенсивный ультразвук нагревает нацеливаемую область до температур около $60^{\circ} \mathrm{C}$. Сразу после лечения пациенты демонстрируют улучшение состояния тремора с минимальными осложнениями [30].

\section{2. МРТ-управляемая радиационная терапия (MR-XRT)}

МРТ-управляемая радиационная (рентгеновскими лучами) терапия (MR-XRT) представляет концепцию объединения MPT-сканера и линейного ускорителя (MR Linac) [31]. Эта технология позволяет одновременно генерировать магнитно-резонансные изображения и доставлять пучки рентгеновского излучения точно к цели, что позволяет проводить лучевую терапию в режиме реального времени. Это особенно эффективно для терапии опухолей, которые меняют свое положение во время дыхания, наполнения мочевого пузыря или сокращения кишечника.

B качестве базовой модели MR Linac выбран линейный ускоритель $6 \mathrm{MB}$ Elekta (Crawley, Великобритания), установленный на кольцевой системе („гентри“), вращающейся вокруг модифицированного $1.5 \mathrm{~T}$ МРТ-сканера „Philips Achieva“ (Best, Нидерланды) (рис. 2).

В линейном ускорителе магнетрон генерирует радиоволны в СВЧ-диапазоне, которые используются для ускорения электронов. Ускоренные до высоких скоростей электроны сталкиваются с твердой металлической мишенью (обычно вольфрам), производя высокоэнергетические рентгеновские лучи. Рентгеновские лучи после фокусировки многолепестковым коллиматором проходят через закрытый канал МРТ-сканера, расположенный в отверстии расщепленного на две части магнита, и затем попадают в опухоль пациента. Эти высокоэнергетические рентгеновские лучи разрушают раковые клетки, вызывая непоправимое повреждение ДНК клеток.

MR-Linac имеет специализированную конструкцию, которая экранирует радиочастотные волны и магнитные поля, создаваемые отдельными частями установки. Применяемые в МРТ-сканере быстрые импульсные последовательности позволяют сканировать интересующие области несколько раз за секунду, что позволяет нивелировать эффекты, связанные с движением тех или иных органов.

MPТ-сканер (1.5 T) обеспечивает не только высококонтрастную визуализацию опухолей в мягких тканях в режиме реального времени, но и отслеживает анатомическое движение опухолей в процессе облучения, что позволяет оптимизировать дозу радиации и место ее доставки, сохраняя при этом окружающие здоровые ткани.

На сегодняшний день доступность гибридных систем MR-Linac ограничена. Проводятся клинические испытания установки, отрабатываются вопросы оптимизации распределения доз в режиме онлайн, надежности процедур сбора информации и отслеживания лечения, интерпретации функциональных маркеров МР-визуализации и их потенциальных изменений в ходе лечения. В процессе постоянного технологического совершенствования применяются как передовые лучевые методы, включая интенсивную модулированную лучевую терапию (IMRT), объемную модулированную дуговую терапию (VMAT) или высокодозную стереотаксическую лучевую терапию тела (SBRT) [32,33], которые преследуют цель доставки высоких доз радиации к опухоли, уменьшая при этом зону риска облучения окружающих опухоль тканей и органов, так и современные методы МРТ-диагностики: технология „Cine MR“ [34] и четырехмерная (4D) $T_{2}$ взвешенная MPT [35].

Кроме того, непрерывно расширяются области применения метода MR-XRT. Возможности MPT-направляемой радиационной (рентгеновскими лучами) терапии опухолей в мягких тканях практически не ограничены. Этот метод апробирован для лечения опухолей головного мозга (метастазы, глиомы, анапластические астроцитомы, олигодендроглиомы, глиобластомы, экстрааксиальные опухоли), шеи (рак ротоглотки), легких (немелкоклеточного рака), молочной железы, желудочно-кишечных опухолей (печени, поджелудочной железы, прямой кишки), опухолей мочеполовой системы (почек, предстательной железы, мочевого пузыря), гинекологических опухолей и различных метастазов [36].

\section{3. МРТ-управляемая протонная терапия (MR-PT)}

Протонная терапия является одним из видов радиационной (корпускулярной) терапии, которая использует протоны, разогнанные в ускорителе частицы, для нацеленного облучения пораженной ткани, причем наиболее часто при терапии раковых заболеваний [37]. Эти ионизирующие частицы повреждают ДНК клеток, вызывая в конечном случае их гибель. Все протоны заданной энергии имеют совершенно определенную глубину проникновения в вещество, лишь ничтожное их количество превышает это расстояние. Практически вся радиационная доза энергии выделяется в ткани на последних миллиметрах пробега частиц (этот максимум потерь энергии радиации называют пиком Брэгга). Местоположение пика Брэгга зависит от энергии, до которой были разогнаны частицы в ускорителе, эта энергия в большинстве случаев находится в диапазоне от 70 до $250 \mathrm{MeV}$. Управление величиной дозы облучения в зависимости от глубины проникновения протонного пучка в ткани дает возможность детерминировать область разрушения больных клеток протонным пучком, не внося повреждений в окружающие здоровые ткани. Благодаря сравнительно большой массе протоны испытывают лишь небольшое поперечное рассеяние в ткани, а разброс дли- 


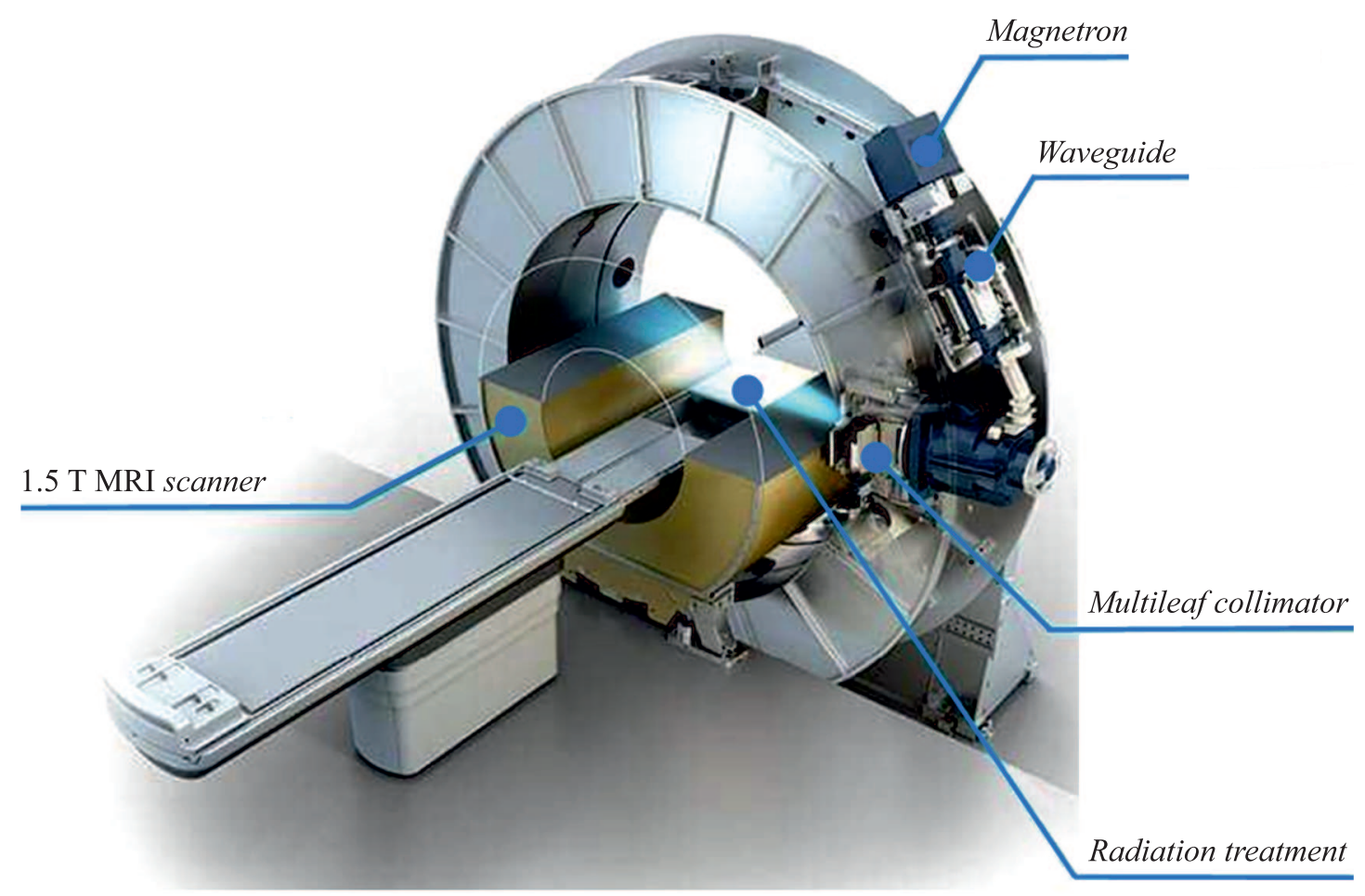

Pис. 2. Система MR-Linac.

ны их пробега очень мал, поэтому область фокусировки протонного пучка может составлять порядка $1 \mathrm{~mm}^{3}$.

Метод протонной лучевой терапии позволяет прецизионно нацеливаться на опухоль и уничтожать ее на любой глубине тела. По этой причине протонная терапия особенно хороша для некоторых видов опухолей, где обычная радиационная терапия наносит неприемлемый ущерб окружающим тканям. Это особенно важно при лечении детей, когда длительное облучение приводит к возникновению вторичных опухолей, возникающих при избыточных радиационных дозах. Из-за меньшей дозовой нагрузки на здоровые ткани протоны создают гораздо меныше побочных радиационных эффектов, чем в обычной радиационной терапии.

Протонная лучевая терапия показала впечатляющие результаты при терапии многих видов рака, включая рак мозга, рак позвоночника, рак простаты. Однако широкомасштабное использование протонных пучков для лечения рака сдерживается размером и стоимостью необходимого ускорительного оборудования. Перспективы развития метода протонной терапии связаны с разработкой компактной ускорительной системы для облучения пациентов протонами и с разработкой концепции сочетания МРТ с протонной лучевой терапией, т. е. MPTуправляемой протонной лучевой терапией (MR-PT) в реальном масштабе времени [38].

Предварительные исследования по объединению МРТ с протонным пучком [39] продемонстрировали, что в принципе этот широко используемый метод визуализации действительно может работать при лечении рака пучком частиц. Многие эксперты предсказывали трудность, возникающую из-за поведения протонного пучка: при движении электрически заряженных частиц в магнитном поле МРТ-сканера силы Лоренца будут отклонять протонный пучок от прямолинейной траектории. Однако исследователи смогли продемонстрировать, что это отклонение можно предвидеть и скорректировать соответствующим образом. С помощью специального экранирования удалось исключить электромагнитные взаимодействия между МРТ-сканером и оборудованием для протонной терапии. В этом исследовании [39] впервые МРТ-сканер со слабым магнитным полем (0.22 Т) был интегрирован с исследовательской линией протонного пучка для проверки возможности одновременного облучения и визуализации. МРТ-изображения тканеподобного фантома были получены в статическом магнитном поле МРТ-сканера в сочетании с динамическими градиентными полями при использовании различных импульсных радиочастотных (РЧ) последовательностей в присутствии и отсутствие облучения протонным пучком. Было показано, что МР-визуализация возможна в ,электромагнитно загрязненной“ среде оборудования протонной терапии. Наблюдаемое качество анатомических МР-изображений оценивалось как достаточное для определения и позиционирования объема мишени. Имитирующий ткань фантом не показывал видимой деградации изображения, вызванной протонным пучком. Никакого существенного влияния динамических градиентных полей и РЧ импульсных последовательностей МРТ-сканера на профили протонных пучков обнаружено 


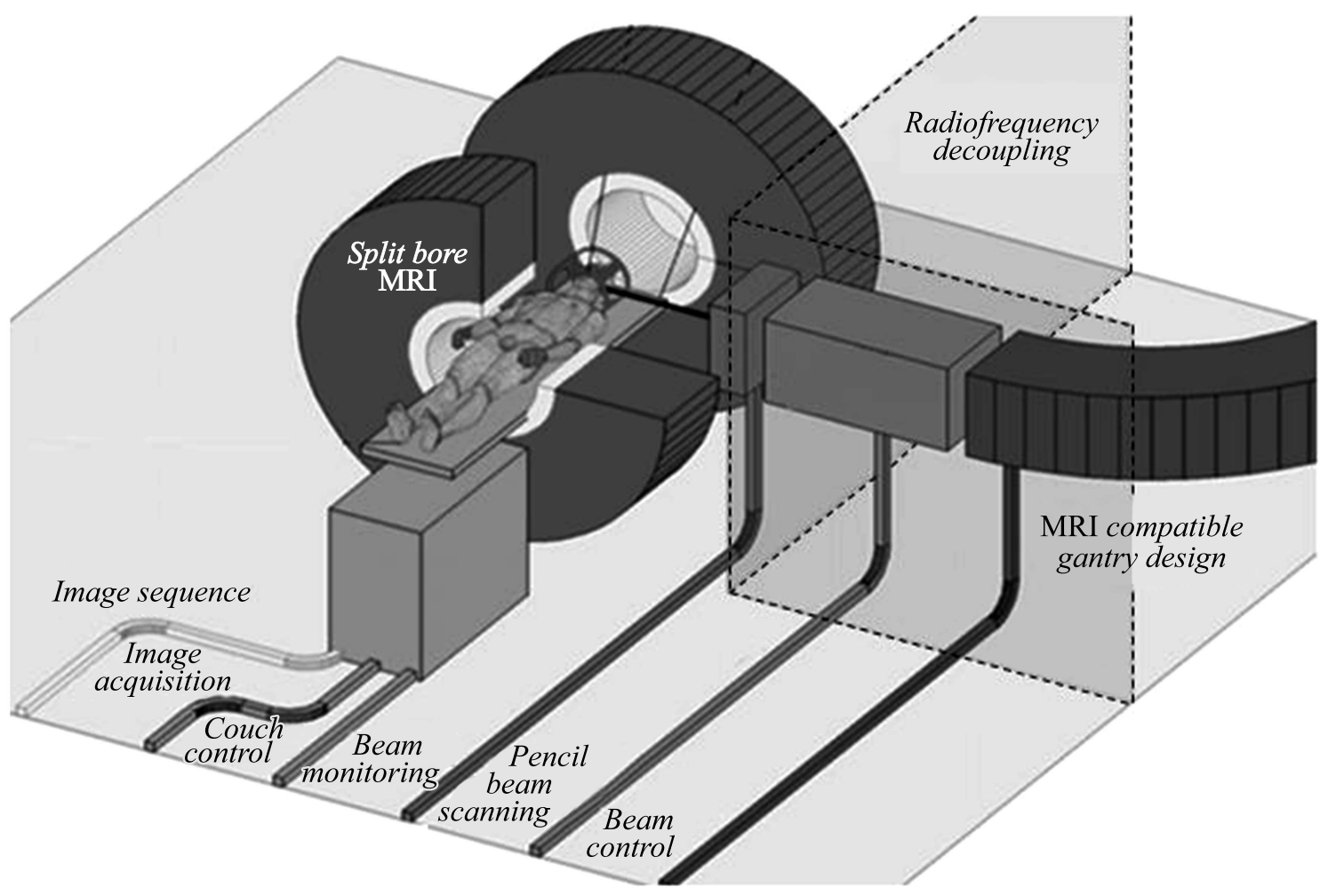

Pис. 3. Прототип системы MR-PT.

не было. Эти исследования доказали, что одновременное облучение пучком и МР-визуализация технически осуществимы с помощью низкопольного МРТ-сканера, интегрированного со статической исследовательской линией протонного пучка.

Использование низкопольного МРТ-сканера позволяет не только исключить взаимовлияние магнитных полей МРТ-сканера и магнитных систем протонной ускорительной системы, ослабить влияние магнитного поля сканера на траекторию движения протонного пучка, но и использовать ряд особенностей ЯМР, проявляющихся именно в слабом магнитном поле и, следовательно, при пониженной частоте. Сюда относится эффект частотной дисперсии времен релаксации: для биологических объектов скорость ЯМР-релаксации в слабом магнитном поле увеличивается на два-три порядка величины. Это, вопервых, позволяет получать изображения повышенной информативности, а во-вторых, частично скомпенсировать потерю чувствительности путем увеличения числа повторений при усреднении сигнала за фиксированный промежуток времени. Кроме того, при использовании слабого магнитного поля на один-два порядка снижается стоимость прибора. Это связано с упрощением конструкции благодаря отсутствию сверхпроводящей системы для создания магнитного поля.

Оценивая возможность использования МРТ в слабых магнитных полях для протонной терапии, следует отметить простоту и гибкость конструкции магнитной системы, что позволяет упростить совмещение МРТ-ска- нера со сложной громоздкой аппаратурой ускорительной техники.

В настоящее время проводятся исследования по инновационным подходам и технологиям с целью создания первого в мире прототипа для МРТ-управляемой протонной терапии, применимого для клинического использования. Рассматриваются различные концепции построения такой системы. Один из прототипов [38] приводится на рис. 3.

Актуальность практической реализации МРТ-управляемой протонной лучевой терапии (MR-PT) обусловлена стремительным ростом числа центров протонной терапии в мире: в 2009 году было 52 таких центра, в конце 2016 года их функционировало уже 162 [40]. Кроме того, возрастает интерес к использованию в медицине методов углеродно-ионной и гелий-ионной терапии.

\section{4. МРТ-управляемая радиочастотная терапия (MR-RFT)}

Исследования в области МРТ-управляемой радиочастотной терапии проводятся по двум основным направлениям:

- МРТ-управляемая радиочастотная гипертермия (MR-RFHT) [41],

- квантовая магнитно-резонансная терапия (QMRT) [42].

Метод МРТ-управляемой радиочастотной гипертермии имеет концепцию, схожую с концепцией метода 


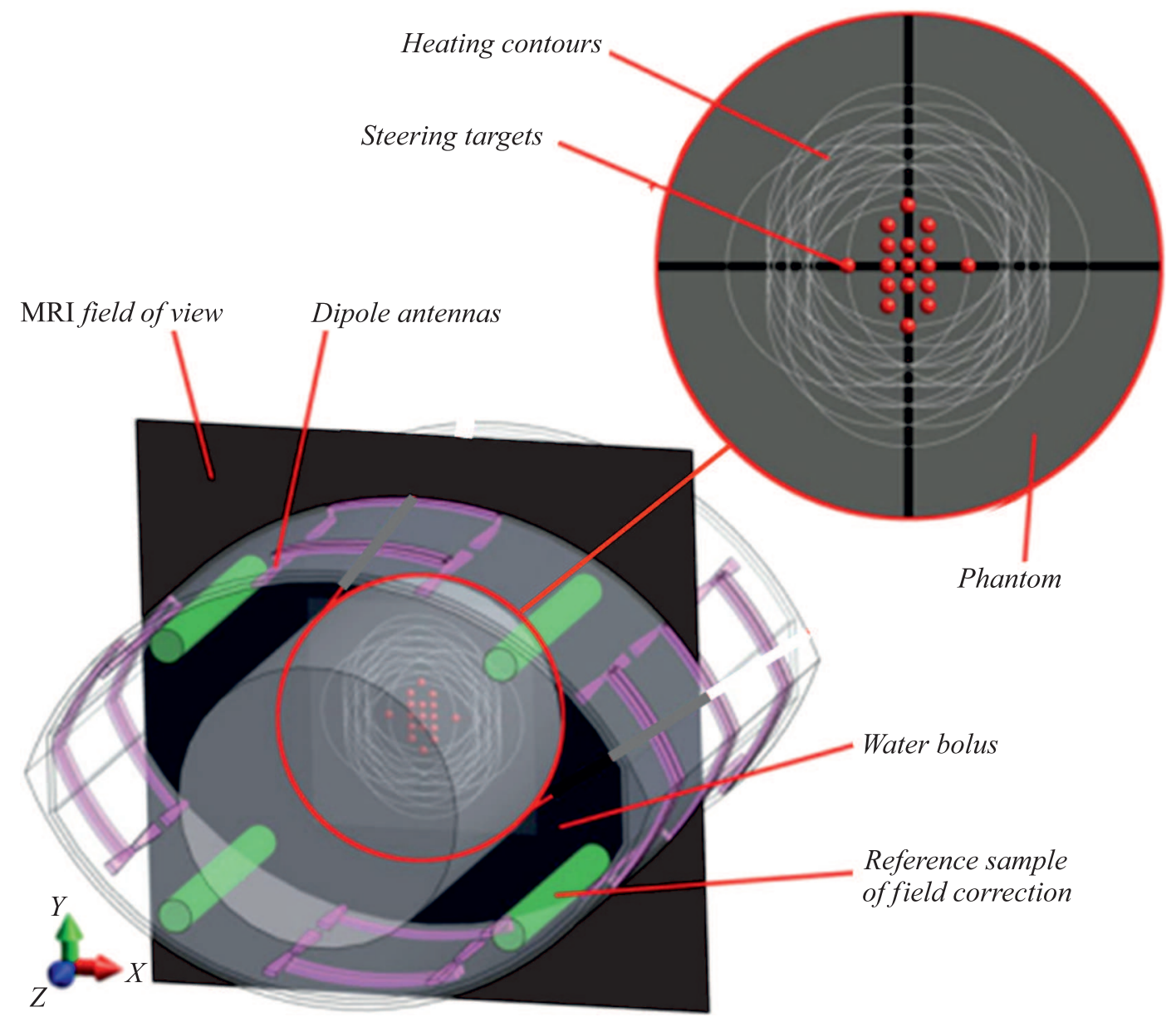

Рис. 4. МРТ-совместимый РЧ аппликатор.

MR-HIFU, однако для селективного нагревания до относительно высоких температур $\left(40-45^{\circ} \mathrm{C}\right)$ пораженных раковыми клетками тканей вместо фокусированного ультразвукового пучка используются фокусированные радиочастотные (РЧ) (высокочастотные) электромагнитные волны. Для того чтобы нагревание было точным и глубоким, но при этом разрушало только раковые клетки, не нанося вреда близлежащим здоровым тканям, процесс тщательно контролируется. Многочисленные исследования доказали, что умеренная $\left(40-45^{\circ} \mathrm{C}\right.$ в течение $60 \mathrm{~min}$ ) РЧ гипертермия (RFHT) как адъювантный метод лечения онкологических пациентов значительно повышает эффективность химиотерапии и лучевой терапии. При этом RFHT практически не имеет побочных эффектов.

Для создания фокусированного РЧ поля используется РЧ аппликатор. Конструкция МРТ-совместимого РЧ аппликатора Pyrexar BSD2000-3D1 [41], также известного как Sigma Eye, имеет эллиптическую форму (рис. 4).

РЧ аппликатор состоит из 24 дипольных антенн, которые расположены в трех кольцах („голова“, „середина“", „ноги“) с четырьмя („сверху“, „снизу“, „слева“ и „справа“
Амплитуду РЧ сигналов $(100 \mathrm{MHz})$ в каждой паре дипольных антенн и область участка РЧ воздействия можно менять независимо друг от друга, что позволяет управлять $3 D$-фокусом электромагнитной энергии. Между антеннами и объектом исследования (фантомом, поперечное сечение которого в верхней правой части рис. 4 выделено серым кругом) находится водяной болюс, который заполнен деионизированной водой. Это позволяет эффективно передавать электромагнитную энергию от антенн к фантому, а также обеспечивать охлаждение поверхности фантома. Аппликатор можно использовать также в качестве РЧ катушки МРТ-сканера (для всего тела). Для коррекции временного дрейфа поля $B_{0}$ используются интегрированные в аппликатор эталонные трубки, заполненные реперным жироподобным веществом.

Черная плоскость на рис. 4 - это поле зрения MPТ-сканера. Точки в центре поперечного сечения фантома являются целями РЧ теплового воздействия, а белые замкнутые линии представляют собой соответствующие идеализированные контуры 50\% нагрева.

Для картирования распределения температуры РЧ нагрева, так же как и в методе MR-HIFU, используется 
метод оценки сдвига частоты протонного резонанса в зависимости от температуры (PRFS или MRTI) [10,43].

Исследования на фантоме по применению аппликатоpa Pyrexar BSD2000-3D, совместимого с 1.5 T МРT-сканером, для целей РЧ гипертермии показали, что этот аппликатор обеспечивает надежный и воспроизводимый нагрев с точностью изменения температуры на $0.2^{\circ} \mathrm{C}$ изза ошибок позиционирования [41].

В основе метода квантовой магнитно-резонансной терапии (QMRT) [42] лежит использование MPT для измерений плотности протонов ткани-мишени с последующим облучением сформированными по специальной программе радиочастотными электромагнитными волнами, нацеленными с помощью МРТ на опухолевые ткани для манипулирования биофизической клеточной сигнализацией. Циркулярно поляризованные безопасные электромагнитные волны с радиочастотой от $30 \mathrm{kHz}$ до $300 \mathrm{MHz}$ могут быть точно ориентированы под контролем и управлением МРТ на целевые ткани для изменения потенциала мембран клеток, что, в свою очередь, может стимулировать рост тканей при дегенеративных заболеваниях, таких как остеоартрит [44], или вызывать апоптоз (программируемую гибель) клеток и влиять на рост плотных опухолей [42]. Потенциал клеточной мембраны $V_{m}$ создается из-за разницы в концентрации ионов внутри и снаружи мембраны клетки и для нормальных клеток составляет от -70 до $-90 \mathrm{mV}$. При злокачественной трансформации нормальных клеток происходит значительная деполяризация мембран клеток, что играет важную роль для появления и поддержания раковых стволовых клеток, которые необходимы для устойчивого роста опухоли [45]. Это объясняет роль потенциала $V_{m}$ в процессе биоэлектрической сигнализации раковых клеток, изменение которого способствует регулированию процессов пролиферации, миграции и дифференциации клеток. C помощью метода QMRT потенциал клеточной мембраны $V_{m}$ может быть „искусственно модифицирован“, для того чтобы замедлить рост опухоли и метастазирование.

Поскольку влияние электромагнитного поля вызывает широкий спектр физиологических воздействий на отдельные типы клеток и тканей [46], были проведены исследования применения метода QMRT для лечения пациентов с такими хроническими дегенеративными заболеваниями, как остеоартрит [44] и рассеянный склероз [42]. Несмотря на положительные результаты, осознание и объяснение эффектов, наблюдаемых при использовании данного метода, для оценки его терапевтических возможностей требуется систематизация данных о влиянии слабого электромагнитного поля на различные органы.

\section{Заключение}

Интерес к МР-тераностике быстро растет. Результаты исследований и разработки в этой области находят все более широкое применение в клинической практике. Подчеркнем, что МРТ выступает не только как средство диагностики, но и в качестве гида, направляющего лечебное воздействие физических полей разнообразной природы. Появляются новые методы МР-тераностики, в которых используются не только ЯМР, но и другие методы магнитного резонанса, такие как электронный парамагнитный резонанс $[2-4,47]$, динамическая поляризация ядер [2-4].

\section{Финансирование работы}

Некоторые аспекты вопросов, обсуждаемых в работе, были изучены в связи с выполнением гранта РФФИ 1853-34003 Куба_т.

\section{Конфликт интересов}

Авторы заявляют, что у них нет конфликта интересов.

\section{Список литературы}

[1] Peter A. Rinck. Magnetic Resonance in Medicine. Blackwell Wissenschafts-Verlag Berlin. Vienna, 2001. 252 p.

[2] Chizhik V.I., Chernyshev Y.S., Donets A.V., Frolov V.V., Komolkin A.V., Shelyapina M.G. Magnetic Resonance and Its Applications. Springer International Publishing, Switzerland, 2014. $785 \mathrm{p}$.

[3] Богачев Ю.В., Драпкин В.З., Князев М.Н., Попечителев Е.П., Разин И.В., Фролов В.В. Магнитно-резонансная томография в слабом магнитном поле. СПб.: Изд-во СПбГЭТУ „ЛЭТИ“, 2012. 260 c.

[4] Богачев Ю.В., Князев М.Н., Марченко Я.Ю., Наумова А.Н., Тютюкин К.В., Фокин В.А., Фролов В.В., Черненко Ю.С. Диагностический магнитный резонанс. СПб.: Изд-во СПбГЭТУ „ЛЭТИ“, 2013. 212 с.

[5] Hartwigsen G., Kassuba T., Siebner H.R. Combining Transcranial Magnetic Stimulation with (f)MRI. In: Ulmer S., Jansen O. (eds). fMRI. Springer, Berlin, Heidelberg, 2010. Ch. 16. P. 155-167. DOI: https://doi.org/10.1007/978-3-54068132-8_16

[6] Gao M., Fan F., Li D., Yu Y., Mao K., Sun T., Qian H., Tao W., Yang $X$. // Biomater. 2017. Vol. 133. P. 165-175.

[7] Ostroverkhov P., Semkina A., Naumenko V., Plotnikova E., Yakubovskaya R., Vodopyanov S., Abakumov A., Majouga A., Grin M., Chekhonin V., Abakumov M. // Pharmaceutics. 2018. Vol. 10. P. 284.

[8] Chen Fang, Miqin Zhang // J. Control. Release. 2010. Vol. 146. N 1. P. 2-5. DOI: 10.1016/j.jconrel.2010.05.013.

[9] Goodfellow F.T., Simchick G.A., Mortensen L.J., Stice S.L., Qun Zhao // Adv. Funct. Mater. 2016. Vol. 26. P. 3899-3915. DOI: $10.1002 / \mathrm{adfm} .201504444$

[10] Edmundson M., Nguyen TK Thanh, Bing Song // Theranostics. 2013. Vol. 3. N 8. P. 573-582. DOI: $10.7150 /$ thno. 5477

[11] Spirou S.V., Basini M., Lascialfari A., Sangregorio C., Innocenti C. // Nanomaterials. 2018. Vol. 8. N 401. P. 1-22. DOI: $10.3390 /$ nano8060401 
[12] Hynynen K., Damianou C., Darkazanli A., Unger E., Schenk J.F. // Ultrasound Med. Biol. 1993. Vol. 19. P. 9192. DOI: 10.1016/03015629(93)90022-G. PMid:8456533

[13] Salomir R., Palussie're J., Vimeux F.C., de Zwart J.A., Quesson B., Gauchet M., Lelong P., Pergrale J., Grenier N., Moonen C.T.W. // J. Magn. Reson. Imaging. 2000. Vol. 12. P. 571-583. DOI: 10.1002/15222586(200010)12:45571::AIDJMRI943.0.CO;2-2. PMid:11042639

[14] Palussie're J., Salomir R., Le Bail B., Fawaz R., Quesson B., Grenier N., Moonen C.T.W. // Magn. Reson. Med. 2003. Vol. 49. P. 89-98.

[15] Mougenot C., Quesson B., de Senneville B.D., de Oliveira P.L., Sprinkhuizen S., Palussie're J., Grenier N., Moonen C.T.W. // Magn. Reson. Med. 2009. Vol. 61. P. 603-614. DOI: $10.1002 / \mathrm{mrm} .21887$. PMid:19097249

[16] Hynynen K., Freund W.R., Cline H.E., Chung A.H., Watkins R.D., Vetro J.P., Jolesz F.A. // Radiographics. 1996. Vol. 16. P. 185-195. PMid:10946699

[17] Rieke V., Butts P.K. // J. Magn. Resonance Imaging. 2008. Vol. 27. P. 376-390. DOI: 10.1002/jmri.21265

[18] Keohler M.O., Mougenot C., Quesson B., Enholm J., Le Bail B., Laurent C., Moonen C.T., Ehnholm G.J. // Med. Phys. 2009. Vol. 36. P. 3521-3535.

[19] Ishihara Y., Calderon A., Watanabe H., Okamoto K., Suzuki Y., Kuroda K., Suzuki Y. // Magn. Resonance Med. 1995. Vol. 34. P. 814-823. DOI: 10.1002/mrm.1910340606

[20] Sapareto S., Dewey $W$. // Int. J. Radiat. Oncol. Biol. Phys. 1984. Vol. 10. P. 787-800. DOI: 10.1016/03603016(84)90379-1. PMid:6547421.

[21] Dewhirst M.W., Viglianti B.L., Lora-Michiels M., Hanson M., Hoopes P.J. // Int. J. Hyperthermia. 2003. Vol. 19. P. 267-294. DOI: 10.1080/0265673031000119006 PMid:12745972.

[22] Yarmolenko P.S., Moon E.J., Landon C., Manzoor A., Hochman D., Viglianti B., Dewhirst M. // Int J. Hyperthermia. 2011. Vol. 27. P. 320-343. DOI: 10.3109/02656736.2010.534527 PMid:21591897.

[23] Damianou C., Hynynen K. // J. Acoust. Soc. Am. 1994. Vol. 95. P. 1641-1649. DOI: 10.1121/1.408550. PMid:8176064.

[24] McDannold N.J., King R.L., Jolesz F.A. // Radiology. 2000. Vol. 216. P. 517-523. PMid:10924580.

[25] Voogt M.J., Trillaud H., Kim Y.S., Mali W.P.Th.M., Barkhausen J., Bartels L.W., Deckers R., Frulio N., Rhim H., Lim H.K., Eckey T., Nieminen H.J., Mougenot C., Keserci B., Soini J., Vaara T., Koehler M.O., Sokka S., Maurice A.A.J. van den Bosch // Eur. Radiol. 2012. Vol. 22. P. 411-417. DOI: 10.1007/s00330-011-2262-8 PMid:21901565.

[26] Le Blang S.D., Hoctor K., Steinberg F.L. // Am. J. Roentgenol. 2010. Vol. 194. P. 274-280. DOI: 10.2214/ AJR.09.2842

[27] Huisman M., van den Bosch M.A. // Cancer Imaging. 2011. Vol. 11. P. S161-S166. DOI: 10.1102/14707330.2011.9041PMid:22180520.

[28] Wijlemans J.W., Bartels L.W., Deckers R., Ries M., Mali W.P.Th.M., Moonen C.T.W., van den Bosch M.A.A.J. // Cancer Imaging. 2012. Vol. 12. N 2. P. 387-394. DOI: $10.1102 / 1470-7330.2012 .9038$

[29] Okada A., Murakami T., Mikami K., Onishi H., Tanigawa N., Marukawa T., Nakamura H. // Magn. Reson. Med. Sci. 2006. Vol. 5. P. $167-171$. DOI: $10.2463 / \mathrm{mrms} .5 .167$ PMid:17139143.

[30] Galkin M.V. // PROBLEMS OF NEUROSURGERY named after N.N. Burdenko. 2016. Vol. 80. N 2. P. 99-108.
[31] Raaymakers B.W., Lagendijk J.J.W., Overweg J., Kok J.G.M., Raaijmakers A.J.E., Kerkhof E.M., van der Put R.W., Meijsing I., Crijns S.P.M., Benedosso F., van Vulpen M., de Graaff C.H.W., Allen J., Brown K.J. // Phys. Med. Biol. 2009. Vol. 54. P. N229-N237. DOI: $10.1088 / 0031-9155 / 54 / 12 / \mathrm{N} 01$

[32] Farber L.A., Speck T. // Cancer therapy and oncology. International J. 2018. Vol. 11. N 3. P. 1-6. DOI: $10.19080 / C T O I J .2018 .11 .555811$

[33] Choudhury A., Budgell G., MacKay R., Falk S., FaivreFinn C., Dubec M., van Herk M., McWillian A. // Clinical Oncology. 2017. Vol. 29. N 10. P. 662-666. DOI: 10.1016/j.clon.2017.04.036

[34] Li Feng, Otazo R., Srichai M.B., Lim R.P., Sodickson D.K., Kim D. // J. Cardiovascular Magn. Res. 2011. Vol. 13 (Suppl. 1). P. 25. DOI: 10.1186/1532-429X-13-S1-P25

[35] Freedman J.N., Collins D.J., Gurney-Champion O.J., McClelland J.R., Nill S., Oelfke U., Leach M.O., Weitscherek A. // Radiotherapy Oncology. 2018. Vol. 129. P. 486-493.

[36] Corradini1 S., Alongi F., Andratschke N., Belka C., Boldrini L., Cellini F., Debus J., Guckenberger M., HörnerRieber J., Lagerwaard F.J., Mazzola R., Palacios M.A., Philippens M.E.P., Raaijmakers C.P.J., Terhaard C.H.J., Valentini V., Niyazi M. // Radiation Oncology. 2019. Vol. 14. N 92. P. 1-12. DOI: 10.1186/s13014-019-1308-y

[37] Allen A.M., Pawlicki T., Lei Dong, Fourkal E., Buyyounouski M., Cengel K., Plastaras J., Bucci M.K., Yock T.I., Bonilla L., Price R., Harris E.E., Konski A.A. // Radiotherapy Oncology. 2012. Vol. 103. N 1. P. 8-11. DOI: $10.1016 /$ j.radonc.2012.02.001

[38] Oborn B.M., Dowdell S., Metcalfe P.E., Crozier S., Mohan R., Keall P.J. // Med. Phys. 2017. Vol. 44. N 8. P. e77-e90. DOI: $10.1002 / \mathrm{mp} .12371$

[39] Schellhammer S.M., Hoffmann A.L., Gantz S., Smeets J., Erik van der Kraaij, Quets S., Pieck S., Karsch L., Pawelke J. // Phys. Medicine and Biology. 2018. Vol. 63. N 23. https://doi.org/10.1088/1361-6560/aaece8

[40] PTCOG. Facilities in operation. 2016.

http://www.ptcog.ch/index.php/facilities-in-operation.

[41] Mulder H.T., Curto S., Paulides M.M., Franckena M., G.C. van Rhoon // Intern. J. Hyperthermia. 2018. P. 1-9. DOI: $10.1080 / 02656736.2018 .1497209$

[42] Kumar R., Augustus M., Nair A.R., Ebner R., Nayar G.S., Kumar R.V. // J. Clin. Exp. Oncol. 2016. Vol. 5. N 2. P. 1-11. http://dx.doi.org/10.4172/2324-9110.1000156

[43] Winter L., Oberacker E., Paul K., Yiyi Ji, Oezerdem C., Ghadjar P., Thieme A., Budach V., Wust P., Niendorf T. // Intern. J. Hyperthermia. 2016. Vol. 32. N 1. P. 63-75. DOI: $10.3109 / 02656736.2015 .1108462$

[44] Vasishta V.G., Kumar R.V., Pinto L.J. // Ind. J. Aerospace Med. 2004. Vol. 48. P. 1-7.

[45] Yang M., Brackenbury W.J. // Front. Physiol. 2013. Vol. 4: Art. 185. P. 1-10. DOI: 10.3389/fphys.2013.00185. eCollection 2013

[46] Adey W.R. // J. Cell Biochem. 1993. Vol. 51. P. 410-416.

[47] Aжсиа Я.И. Медико-биологические аспекты применения метода электронного парамагнитного резонанса. М.: Наука, 1983. 528 с. 